\title{
G

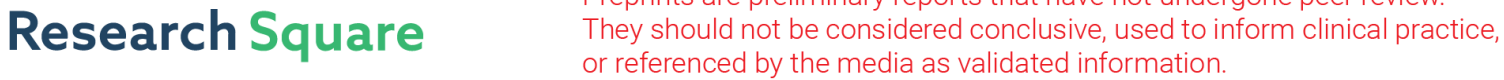 \\ PTH2R Is Related to Cell Proliferation and Migration in Ovarian Cancer: A Multi-omics Analysis of Bioinformatics and Experiments
}

\section{Wang Xiaowei}

First Affiliated Hospital of Harbin Medical University

\section{Lu Tong}

Harbin Medical University Second Affiliated Hospital Department of Cardiology

Qu Yanjun

Harbin Medical University

Lili Fan ( $\nabla$ 102337@hrbmu.edu.cn )

Harbin Medical University https://orcid.org/0000-0002-1318-7017

\section{Primary research}

Keywords: Ovarian cancer, PTH2R, TCGA, GEO, Multi-omics

Posted Date: November 23rd, 2021

DOI: https://doi.org/10.21203/rs.3.rs-1026578/v1

License: (c) (i) This work is licensed under a Creative Commons Attribution 4.0 International License. Read Full License

Version of Record: A version of this preprint was published at Cancer Cell International on April 11th, 2022. See the published version at https://doi.org/10.1186/s12935-022-02566-2. 


\section{Abstract \\ Background}

Ovarian cancer is a common gynecological disease and seriously endangers women's health. Currently, there is still a lack of effective molecular markers for the diagnosis and treatment of ovarian cancer. The present study aimed to investigate the molecular markers associated with ovarian cancer.

\section{Methods}

The molecular and gene related to ovarian cancer were extracted from GEO database and TCGA database by bioinformatics, and the related genes and functions were further analyzed. The results were verified by qPCR, WB, CCK-8 and Transwell experiments.

\section{Results}

Data analysis showed that PTH2R gene was highly expressed in tumors, and 51 HUB genes were obtained. Finally, experimental verification showed that PTH2R gene was highly expressed in ovarian cancer, and PTH2R gene was involved in the proliferation, invasion and metastasis of ovarian cancer cells.

\section{Conclusions}

After experimental verification, we found that knocking down the expression of PTH2R can inhibit the proliferation, invasion and migration of tumor cells.PTH2R is expected to become a new molecular marker for ovarian cancer.

\section{Background}

Ovarian cancer is a malignant tumor that grows on the ovaries. Of all cases, $90-95 \%$ are primary ovarian cancer, whereas 5-10\% comprise metastatic tumors originating from other organs. [1-3] Although ovarian cancer is less common than either cervical or endometrial cancers, its mortality rate exceeds that of cervical and endometrial cancers combined. $[4,5]$ The 5 -year survival rate for ovarian cancer is $<40 \%$, making it one of the deadliest gynecological tumors. [6] Its lethality mainly arises from its aggressive nature and from the difficulty of achieving early diagnosis. As a result, most patients develop highly metastatic, invasive disease in later stages.[7] Ovarian cancer is highly heterogeneous and adenocarcinoma accounts for the majority of malignant tumors. Current treatment strategies include platinum- and taxane-based chemotherapy, as well as neoadjuvant chemotherapy after surgical resection. Unfortunately, most patients relapse or develop drug resistance within 36 months. [8] At 
present, the molecular etiology of the ovarian cancer remains elusive; thus, finding effective biomarkers for the diagnosis and treatment of ovarian cancer is a priority.

In recent years, with the development of sequencing technology, bioinformatics has come to play an important role in revealing the occurrence and development of tumors. $[9,10]$ In the past, many researchers did not have direct access to sequencing data, due to sample or funding constraints. Now, however, an increasing number of researchers are uploading their microarray or sequencing data to public databases, allowing oncology-related researchers around the world to download and process data to reveal the underlying pathogenesis of tumors. Among these, the Gene Expression Omnibus (GEO) [11] and The Cancer Genome Atlas (TCGA) databases [12] are the most widely used. The GEO database contains raw microarray and sequencing data uploaded by numerous researchers, as well as data from a variety of molecular types, including mutation, messenger ribonucleic acid (mRNA), non-coding RNA, and other transcriptome and methylation data. A large number of studies have analyzed public data from GEO; many have used multi-data set joint analysis to find important regulatory molecules. For instance, $\mathrm{Bi}$ et al. used GSE17260 and GSE73614 to develop a glycolysis-related prognostic signature in ovarian cancer. [13] In addition, Jin et al. identified CXCL10 gene as promising biomarker for ovarian cancer immunotherapy. [14] TGCA database is also widely used in oncology studies; it is a pan-cancer project hosted by the National Institutes of Health $(\mathrm{NIH})$, providing a wide variety of tumors and different molecular data types that can be downloaded and analyzed. In the past few years, most studies have focused on RNA sequencing (RNA-Seq) analysis, such as mRNA and non-coding RNAs. [15-17] Recently, following the updating of old algorithms and the discovery of new ones, CIBERSORT, MCPcounter, and other algorithms have been used to assess tumor immune cell infiltration. Meanwhile, ESTIMATE has been used to assess the scores of Immune and stromal cells in the tumor microenvironment. [18-20]

Exploring public databases has revealed many molecules that are highly expressed in ovarian cancer, such as CXCL10 [14], MMP16 [21], MCUR1 [22], MRPL15 [23], et al. In combination with multiple data sets, the expression of the gene Parathyroid Hormone 2 Receptor (PTH2R) has been shown to be significantly elevated in ovarian cancer. To further mine PTH2R-related data, here relevant information was classified into high or low expression PTH2R groups, according to the expression value of PTH2R. Further analyses of these groups, such as mutation characteristics, copy number variation (CNV), drug resistance characteristics, and immune infiltration, were conducted to explore PTH2R-related genes and functions from the perspective of various data. Furthermore, quantitative polymerase chain reaction (qPCR), western blot (WB), Cell Counting Kit 8 (CCK-8), and transwell assays were conducted alongside other experiments to verify the expression and function of PTH2R. Bioinformatics analysis and experimental verification revealed that the high expression of PTH2R can promote the growth, invasion, and metastasis of ovarian cancer. PTH2R may therefore be useful as a potential biomarker for ovarian cancer in the future.

\section{Materials And Methods}

\section{Sample Source}


Ovarian cancer tissue $(n=12)$ and normal ovarian tissue $(n=12)$ samples were collected from gynecology department in The First Affiliated Hospital of Harbin Medical University, from 3 month to 9 month in 2020. None of the patients were treated before undergoing surgery. The surgically resected specimens were immediately placed in liquid nitrogen and then transferred to a $-80^{\circ} \mathrm{C}$ refrigerator for storage. All patients in the study provided written informed consent for the biological study. The research protocol (including specimen collection) was reviewed and approved by the Biomedical Ethics Committee of The First Affiliated Hospital of Harbin Medical University (Batch Number: 20200159), All procedures were conducted in accordance with the Guidelines of the World Medical Association Declaration of Helsinki. The clinicopathological staging and typing of the patients met the Joint Council on Cancer (AJCC) typing criteria.

\section{Public Data Acquisition and Preprocessing}

Using R software (Version 4.1.0, http://r-project.org/) the "GEOquery" package [24] from the GEO database (https://www.ncbi.nlm.nih.gov/geo/) was applied to download the GSE18520 and GSE66957 ovarian expression datasets. The samples in these datasets were sourced from Homo sapiens, and the platform is based on the GPL570 (HG-U133_Plus_2) Affymetrix Human Genome U133 Plus 2.0 Array. The GSE18520 dataset includes 63 samples from 53 ovarian cancer patients and 10 normal samples. GSE66957 includes 57 samples and 12 Normal-ovarian samples from 69 ovarian cancer patients within the dataset. All these data were included in this study.

In addition, count data of ovarian cancer RNA-Seq, single nucleotide polymorphism (SNP) data, and matching clinical data $(n=379)$ were downloaded from TCGA database using Genomic Data Commons (GDC) software (https://portal.gdc.cancer.gov/projects/). As there is no normal control for ovarian cancer in TCGA, here the obtained TCGA data were combined with GTEx to obtain normal ovarian control download samples $(n=88)$ and ovarian cancer samples $(n=427)$. RNA-Seq count data were obtained through the University of California Santa Cruz (UCSC) Xena browser (https://xenabrowser.net/datapages/; the data were corrected in batches).

\section{Screening of Differentially Expressed Genes (DEGs)}

The differentially expressed genes (DEGs) of the GSE118520 dataset were downloaded through the R package "limma" [25], following which the package "ggplot2" was used to draw a volcano map of the DEGs to show their differential expressions. DEGs were considered significant when they met the thresholds of $P<0.05$ and | log2FoldChange | $>1$. Subsequently, DEGs in ovarian cancer and normal samples in the combined TCGA-GTEx dataset were screened using the R package "Deseq2" [26], using the same thresholds as detailed above. Taking the intersections of the DEGs obtained from the two data sets, the candidate gene of interest was then selected for subsequent analysis.

\section{Mutation and CNV Analysis}

The somatic mutation data of TCGA-OV patients were extracted by using the R package "maftools" [27]. Somatic mutation data of patients in the high and low gene expression groups were then collected and 
analyzed.

To analyze the changes in CNVs in TCGA-OV patients within the high gene expression group, the $\mathrm{R}$ package "TCGAbiolinks" [28] was used to download the "Masked Copy Number Segment" data of patients. GISTIC 2.0 analysis of the downloaded CNV fragments was then conducted through GenePattern (https://cloud.genepattern.org) [29].

\section{Weighted Gene Co-expression Network Analysis (WGCNA)}

The R package "WGCNA" [30] was used to analyze the GSE18520 and TCGA-OV datasets. The samples were divided into high and PTH2R low expression groups. The standardized data were then used to construct a co-expression network. For all functions in WGCNA, the correlations of double weights were used as the correlation method. A topological overlap metric (TOM) was used for network construction and module identification. The calculation parameters minModuleSize $=50$ and mergeCutHeight $=1,000$ were used to analyze data. Ultimately, the hub genes were obtained from the intersection of the genes in the module with the highest significance, and using the previously obtained DEGs.

\section{Functional Enrichment Analysis}

Gene ontology (GO) analysis is commonly used to conduct large-scale functional enrichment studies, including biological process (BP), molecular function (MF), and cellular component (CC) [31]. The Kyoto Encyclopedia of Genes and Genomes (KEGG) is a widely used database that stores information about genomes, biological pathways, diseases, and drugs [32]. Here, GO annotation and KEGG pathway enrichment analyses were performed on the hub gene using the R package "clusterProfiler" [33]. A critical value of false discovery rate $(F D R)<0.05$ was considered to imply statistical significance.

Gene Set Enrichment Analysis (GSEA) is a calculation that analyzes whether a particular set of genes is statistically different between two biological states. It is commonly used to estimate changes in the activities of pathways and biological processes in sample expression datasets. Here, GSEA was conducted to study the differences in biological processes between groups based on the gene expression profile data set of TCGA-OV patients [34]. The gene set "c2.cp.kegg.v7.2.symbols" was downloaded from the MSigDB database [35] for GSEA, and FDR $<0.25$ and $P<0.05$ were considered to represent a significant enrichment.

\section{Drug Sensitivity Analysis}

The CellMiner database (https://discover.nci.nih.gov/cellminer/) is a web-based tool that contains genomic and pharmacological information for researchers to use transcripts and drug response data from the $\mathrm{NCl}-60$ cell line [36]. The data were compiled by the National Cancer Institute. CellMiner provides transcriptional expression levels for the drug responses of 22,379 genes, 360 microRNAs, and 20,503 compounds [37]. The mRNA expression profiles and drug activity data including the PTH2R gene were downloaded from the CellMiner database. The correlation between PTH2R gene expression and compound sensitivity was calculated through Pearson's correlation analysis. $P<0.05$ was considered to represent statistical significance. 
The Genomics of Drug Sensitivity in Cancer (GDSC) database(www.cancerrxgene.org/)can be used to search for tumor drug response data and genome sensitive markers [38]. Here, the pRRophetic algorithm [39], the ridge regression model, and IC50 were used to predict the sensitivities of the high and low PTH2R expression groups to common anticancer drugs.

\section{Immune Cell Infiltration Analysis and Tumor Immunoanalysis}

CIBERSORT (http://CIBERSORT.stanford.edu/) and the LM22 characteristic gene matrix were used to predict the proportions of 22 immune cells in all samples within the predicted dataset [40]. CIBERSORT was used to assess the abundances of 22 immune cells in TCGA-OV dataset, and to calculate the correlations between these 22 kinds of immune cells. Then, by integrating candidate gene expression, Pearson's correlations were calculated between the gene expression and these immune-infiltrating cells, with $P<0.05$ being considered to represent statistical significance.

\section{Cell Culture}

The IOSE-80, SK-OV-3, and A2780 cell lines were bought from the American Type Culture Collection (Manassas, VA, USA). All cells were cultured in high glucose Dulbecco's modified Eagle's Medium (DMEM, Corning) treated with $10 \%$ fetal bovine serum (FBS; Hyclone) and $1 \%$ penicillin/streptomycin solution (Invitrogen) in a $37^{\circ} \mathrm{C}$ humidity, $5 \% \mathrm{CO}_{2}$ incubator.

\section{Real-time Fluorescence qPCR}

Total RNA was extracted using RNAiso Plus reagent (Takara Bio, Kusatsu, Japan). RNA concentration and purity were assessed using a NanoDrop 2000 system (Thermo Fisher, Carlsbad, CA, USA). Reverse transcription was then performed using the PrimeScript ${ }^{\text {Tm }}$ RT reagent kit with gDNA eraser (Perfect Real Time; Takara Bio). The SYBR ${ }^{\circledR}$ Premix Ex AQ ${ }^{\mathrm{T}}$ II (Tli RNaseH Plus; Takara Bio) in ABI 7500 Fast System (Life Technologies, Carlsbad, CA, USA) was used for real-time qPCR; Primers 5'GAGGAACAGTGGGGAAAATATCG -3' (Forward) and 5'- TGGGGTTACAGTGTCGGAAAG' (Reverse) were used for amplification of the entire human PTH2R coding sequence (GenBank accession number NM_005048), sequences used for human GAPDH were GGAGCGAGATCCCTCCAAAAT -3' (Forward) and 5'- GGCTGTTGTCATACTTCTCATGG' -3' (Reverse). The $2^{-\triangle \Delta C t}$ method was used to calculate gene expressions.

\section{Plasmid Transfection}

The PTH2R gene was amplified from HEK293T by standard PCR and then subcloned into pcDNA3.1-HA vector. All plasmids were sequenced. Lipofectamine 2000 (Invitrogen) was used for transfection, according to manufacturer's instructions.

\section{Cell Proliferation Detection}

The CCK-8 assay (CCK-8 SAB Biotech. College Park, MD, USA) was used to detect cell proliferation. According to the manufacturer's protocols, the cells were seeded into six-well plates at a density of $1.0 \times$ 
$10^{5}$ cells per well, and were then cultured in medium supplemented with $5 \%$ FBS for $24 \mathrm{~h}$ (at $37^{\circ} \mathrm{C}$ and $5 \%$ $\mathrm{CO}_{2}$ ). Then, $24 \mathrm{~h}$ after transfection, the cells were digested with trypsin and inoculated in triplicate into 96well plates $\left(3 \times 10^{4}\right.$ cells per well). Each well was incubated with $10 \mu \mathrm{L} /$ well CCK- 8 solution for $2 \mathrm{~h}$ every day, for a total of $5 \mathrm{~d}$. The optical density at $450 \mathrm{~nm}$ was measured on a microplate reader. Three independent replications were performed.

\section{Transwell Invasion and Migration Experiments}

Transwell experiments were divided into transwell migration and transwell invasion experiments. The basic operations were as follows: transwell cells were placed into a 24-well culture plate, the chamber is referred to herein as the superior chamber and the culture plate is referred to as the lower compartment. The cells were then digested in a serum-free medium, following which the cell density was adjusted to $1 \times$ $10^{6} \mathrm{cell} \mathrm{s} / \mathrm{mL}$ and the sample was inoculated in the upper chamber. Dulbecco's Modified Eagle Medium (DMEM) containing 10\% FBS was then added to the lower chamber. Transwell invasion assays were performed by precoating the upper membrane with $40 \mu \mathrm{L}$ of matrix glue (BD Biosciences, USA); the cells were fixed with $4 \%$ paraformaldehyde and were washed with phosphate buffer solution (PBS) after $24 \mathrm{~h}$. Then, they were stained with $0.1 \%$ crystal violet (Solarbio, China) and representative images were observed at x100 and x200 magnification with an optical microscope (Olympus, Tokyo, Japan).

\section{Clone Formation Experiment}

The cells were digested with $0.25 \%$ trypsin until individual cells were obtained; the cell suspension was then diluted to a concentration of $1 \times 10^{4}$ cells $/ \mathrm{mL}$. Then, 1,500 cells from the medium were added to each well of a six-well plate and incubated at $37^{\circ} \mathrm{C}$ in $5 \% \mathrm{CO}_{2}$. When the clones are visible to the naked eye in the six-well plate, cell culture was stopped and the cells were fixed in $4 \%$ paraformaldehyde for 15 minutes. Crystal violet staining was then performed, and the number of clones for which there were more than 50 cells was counted using an optical microscope.

\section{Immunohistochemical Test}

Immunohistochemistry (IHC) was conducted according to the antibody supplier's instructions. Sections of clinical samples were incubated overnight at $4^{\circ} \mathrm{C}$ with a PTH2R primary antibody at different dilution ratios. Images were captured at appropriate magnification under an optical microscope (Nikon Microsystems, Shanghai, China). The antibody used in this study was anti-PTH2R (Chemicon International and GenWay Biotech).

\section{Western Blot}

Total cellular proteins were extracted using radioimmunoprecipitation assay (RIPA) lysates (Beyotime, Shanghai, China). Proteins were isolated and transferred to polyvinylidene fluoride (PVDF) membranes (Millipore, Temecula, CA, USA) by using $7.5 \%$ or $10 \%$ sodium dodecyl sulfate-polyacrylamide gel electrophoresis (SDS-PAGE). The membrane was sealed with primary resistance to PTH2R (Chemicon International and GenWay Biotech) at $4^{\circ} \mathrm{C}$ overnight, following which the membrane was then washed 
and incubated with secondary antibodies. Protein bands were detected using enhanced chemiluminescence (Thermo Scientific Carlsbad, CA, USA).

\section{Statistical Analysis}

All data processing and analysis were completed by $\mathrm{R}$ software (version 4.0.2). For the comparison of the two groups of continuous variables, the statistical significance of the normally distributed variables was estimated using the independent Student $t$ test, and the differences between the non-normally distributed variables were analyzed using the Mann-Whitney $U$ test (i.e. Wilcoxon rank-sum test). The Chi-square test or Fisher's exact test was used to compare and analyze the statistical significance between the two groups of categorical variables. $\mathrm{P}<0.05$ was considered statistically significant.

\section{Results}

\section{High Expression of PTH2R Gene in Tumors}

The work flow is shown in Figure 1. Screening and sorting of ovarian cancer data from the GEO database and sequencing data from TCGA (combined with the GTEx dataset) revealed that PTH2R was differentially expressed in the GSE18520, GSE66957 and TCGA-OV datasets; it was significantly highly expresses in tumor tissue (Figure 2A-2C). PTH2R, which is also known as PTHR2, has recently been found to regulate intracellular calcium and influence keratinocyte differentiation [41]. To date, PTH2R has not been studied in ovarian cancer, however, so here PTH2R was chosen as the study object. The function of PTH2R was explored and verified through subsequent analyses and experiments.

ROC analysis showed that the samples of the two datasets (GSE18520 and TGCA-GTEx) could be better distinguished after grouping them according to the expression of PTH2R (Figure1D, E). Comparing the DEGs in the high and low PTH2R expression groups revealed that the 2,448 genes were differentially expressed in GSE1850, while 1,984 DEGs found in TCGA-OV (Figure 2F-2I).

\section{Mutation and Copy Number Variation Analysis of PTH2R}

In the high PTH2R expression group, significant mutations were observed in the TP53, TTN, CSMD3, AHANK and DNAH10 genes; they were 96\%, 24\%, 12\%, 6\% and 6\%, respectively. In the low PTH2R expression group, significant mutations were observed in the TP53, TTN, MUC16, BRCA1and FAT3 genes; they were $87 \%, 22 \%, 10 \%, 7 \%$ and $7 \%$ (Figure 3A-3B). Based on the mutation sites of PTH2R, the mutation information could be plotted (Figure 3C).

In addition, by collating CNV information of TCGA-OV, it was possible to calculate CNV changes in the high PTH2R expression group (using GISTIC 2.0; Figure 3D), revealing that the 3q26.2, 5q13.2, 8q24.21 and 19p13.3 locus of said group changed significantly.

\section{WGCNA}


By associating module feature genes with grouping information for both datasets, WGCNA revealed that 9 feature modules were determined in TCGA-OV. There were 3 modules with significant positive correlations and 6 modules with significant negative correlations (Figure 4A, 4B). Furthermore, 11 feature modules were determined in GSE18520: there were 3 modules with significant positive correlations and 8 modules with significant negative correlations (Figure 4D, 4E). The greater the correlation coefficient, the greater the correlation with PTH2R expression. Subsequently, the MEpink module with the largest correlation coefficient in TCGA-OV and the MEbrown module with the largest correlation coefficient in TCGA-OV were selected, as shown in Figure 4C and 4F.

\section{Functional Enrichment Analysis}

Intersecting the genes in the module with the previously obtained DEGs resulted in the identification of 51 hub genes. Functional enrichment analysis was conducted for these hub genes, with GO revealing that the DEGs were closely related to the detection of chemical stimulus involved in sensory perception of smell, sensory perception of smell, detection of chemical stimulus involved in sensory perception and snRNA 3'-end processing biological processes (Figure 5B). KEGG functional analysis indicated that the differentially expressed genes mainly affected the Pathogenic Escherichia coli infection, Cardiac muscle contraction, Olfactory transduction and Amyotrophic lateral sclerosis pathways. (Figure 5C) The GSEA results showed that REACTOME_ANTIMICROBIAL_PEPTIDES,

REACTOME_NEUTROPHIL_DEGRANULATION, REACTOME_INTERLEUKIN_10_SIGNALING et al. pathways were significantly enriched in high PTH2R group, while

REACTOME_CLASS_C_3_METABOTROPIC_GLUTAMATE_PHEROMONE_RECEPTORS, REACTOME_GLUCURONIDATION et al. were mainly enriched in low PTH2R group. (Figure 5D, 5E)

\section{Drug Sensitivity Analysis and Drug Prediction}

Sorted by relevance, the top eight drugs most associated with PTH2R were selected. As shown in Figure 6A, PTH2R was negatively correlated with Epothilone B, Alvespimycin, Tanespimycin, geldanamycin analog, Actinomycin D, Mithramycin, Depsipeptide and Pelitrexol.

In addition, IC50 value analysis of the high and low expression PTH2R groups using the GDSC database revealed that common anti-tumor drugs such as Docetaxel, Gefitinib had no significant difference, whereas Cisplatin, Lapatinib et al. did have a difference (Figure 6B).

\section{Effect of PTH2R Gene on Immune Cell Infiltration in TCGA- OV Patients}

To analyze the relationship between PTH2R gene expression and immune cell infiltration in TCGA-OV microenvironment, the proportion of immune cell invasion in the tumor microenvironment was calculated using the CIBERSORT algorithm. Figure 7A and 7B present landscape of immune cell infiltration in TCGA$\mathrm{OV}$ tumor microenvironment and the correlation results of the immune cell score, respectively. PTH2R was found to be significantly positively correlated with Plasma cells, $T$ cells follicular helper, Eosinophils et al. and negatively correlated with Dentritic cells resting, Neutrophils, NK cells activated. 
Moreover, the comparison between high and low PTH2R expression group on TMB and MSI showed that TMB was significantly increased in the high PTH2R expression group ( $p=0.013$ ), while the MSI showed no significant difference. (Figure 7D and 7E)

\section{PTH2R Gene Expression Verification}

Comparing the PTH2R gene expression levels in ovarian cancer and normal tissues, and in the ovarian cancer cells and normal ovarian epithelial cells, revealed that the expression of PTH2R was significantly higher in ovarian cancer tissues and cells than in normal ovarian tissues and cells (Figure 8A and 8B). Subsequently, western blot and IHC results also showed that PTH2R protein expression was significantly higher in tumor tissues than in normal tissues (Figure 8C and 8D); this result is consistent with the expression trend of PTH2R in RNA-Seq data observed in the previous database.

\section{Inhibition of Proliferation, Invasion, and Metastasis of Ovarian Cancer Cells by PTH2R Knockdown}

The proliferation ability of PTH2R on ovarian cancer cells was detected by CCK-8 assay, revealing that compared with the control group, the decreased expression of PTH2R reduced the A2780 and SKOV3 proliferation activity (Figure 9A and 9B). Similarly, the colony formation assay revealed that the downregulation of PTH2R inhibited the colony formation of A2780 and SKOV3 cells, compared with the control group (Figure 9C). Subsequently, transwell invasion and migration experiments further demonstrated that PTH2R downregulation significantly reduced the invasion and migration of tumor cells (Figure 9D and 9E). In conclusion, the PTH2R gene was found to be involved in the proliferation, invasion, and metastasis of ovarian cancer. This result further validates the results of the bioinformatical analysis.

\section{Discussion}

Ovarian cancer is the most malignant cancer among diseases of the female reproductive system, accounting for more than $90 \%$ of ovarian cancer deaths. If epithelial ovarian cancer is identified at stage II or III, the estimated 5-year mortality rate is 70\%. [42] Currently, the standard treatment for patients with advanced ovarian cancer comprises cytolytic surgery and postoperative chemotherapy. However, the efficacy and prognosis of ovarian cancer patients remain poor due to poor tolerance to chemotherapy and the lack of effective monitoring measures. Thus, it is expected that new targets can be found for the diagnosis and treatment of ovarian cancer.

With the current progress in bioinformatics and the development of sequencing technology, an increasing number of researchers are gaining access to larger sample sets from public databases from which to mine valid information. In this study, the combined TCGA-OV data set and the GSE18520, GSE66957 data sets showed that PTH2R was significantly elevated in ovarian cancer tissues. Tumor samples were further divided into high and low PTH2R expression groups, which allowed PTH2R-related genes and molecular characteristics to be better investigated. Analyzing the mutation characteristics of the high and low PTH2R expression groups revealed that there were significant mutation differences between the two. 
In the high PTH2R expression group, the CSMD3, AHANK, CSMD1 et al. mutations were more frequent, while in the low expression group, the MUC16, BRCA1, FAT1 et al. mutations were more frequent. In addition, CNV changes were also identified in high PTH2R expression group, and significant changes were identified at 3q26.2, 5q13.2, 8q24.21 and 19p13.3 locus. Analysis of drug resistance revealed that resistance to Cisplatin and Imatinib was more obvious in the high PTH2R expression group.

Moreover, with the development of immunotherapy, here the relationship between PTH2R and immune cell infiltration was investigated, alongside the prospect of immunotherapy, by combining RNA-Seq data and a widely used immune cell scoring algorithm. Comparing the correlations between the expression of PTH2R and immune cell infiltration revealed that PTH2R showed a significant positive correlation with Plasma cells, but was negatively correlated with Dendritic cells resting. However, the coefficient of association was relatively low, so this does not necessarily mean that PTH2R is definitely related to immune response; it also may be due to insufficient of the sample size. In addition, TMB and MSI scores showed that the TMB was significantly increased in the high PTH2R expression group, which may be a potential diagnostic marker.

Although bioinformatics analysis provided important information in this PTH2R study, experiments were still needed to verify the expression and function of PTH2R. Therefore, the mRNA expression and protein levels of PTH2R were investigated through qPCR, WB, and IHC, revealing that it was consistent with the expression trend identified in the databases. In other words, the expression of PTH2R in tumors was significantly higher than that in normal tissues and cells. In addition, a series of tumor cell function experiments, such as CCK-8, clonogenesis, and transwell assays, showed that PTH2R knockdown significantly inhibited the growth, invasion, and migration of tumor cells. In conclusion, PTH2R is expected to become a new molecular marker for ovarian cancer.

This study is the first to report the expression and function of PTH2R in ovarian cancer. Combined with molecular information available in public databases, this study deeply explored the function and mechanism of PTH2R from the perspective of multiple omics; its findings provide an important preliminary basis for future PTH2R-related research. However, there are some limitations in this study, as only the expression and biological function of PTH2R were verified; it was not possible to completely characterize the influences of drug resistance and mutation characteristics.

\section{Conclusion}

In this study, PTH2R was found to be highly expressed in ovarian cancer through bioinformatics methods, combined with the GEO and TCGA databases. Experiments verified that PTH2R was not only highly expressed in tumor cells and tissues, but also affected the proliferation, invasion, and migration of tumor cells. PTH2R is thus expected to become a new molecular marker for ovarian cancer.

\section{Abbreviations}


BP, biological process, CCK-8, Cell Counting Kit 8, CC, cellular component, CNV, copy number variation, DEGs, Differentially Expressed Genes, DMEM, Dulbecco's Modified Eagle Medium, FDR, false discovery rate, GEO, Gene Expression Omnibus, GO, Gene ontology, GSEA, Gene Set Enrichment Analysis, GDC, Genomic Data Commons, GDSC, Genomics of Drug Sensitivity in Cancer, IHC, Immunohistochemistry, KEGG, Kyoto Encyclopedia of Genes and Genomes, mRNA, messenger ribonucleic acid, MSI, microsatellite instability, MF, molecular function, PTH2R, Parathyroid Hormone 2 Receptor, PBS, phosphate buffer solution, PVDF, polyvinylidene fluoride, qPCR, quantitative polymerase chain reaction, RIPA, radioimmunoprecipitation assay, ROC, receiver operating characteristic, RNA-Seq, RNA sequencing, TCGA, The Cancer Genome Atlas, TOM, topological overlap metric, TMB, tumor mutation burden, WGCNA, Weighted Gene Co-expression Network Analysis, WB, western blot

\section{Declarations}

\section{Ethics approval and consent to participate}

All patients in the study provided written informed consent for the biological study. The research protocol (including specimen collection) was reviewed and approved by the Biomedical Ethics Committee of The First Affiliated Hospital of Harbin Medical University Hospital (Batch Number: 20200159). All procedures were conducted in accordance with the Guidelines of the World Medical Association Declaration of Helsinki. The clinicopathological staging and typing of the patients met the AJCC typing criteria.

\section{Consent for publication}

Not applicable.

\section{Availability of data and materials}

The datasets used and/or analyzed during this study can be acquired from the corresponding author upon reasonable request.

\section{Competing interests}

The authors declare that they have no competing interests.

\section{Funding}

None.

\section{Authors' contributions}

WXW and FLL participated in the design of this study, LT performed the study, analyzed this study and drafted the manuscript. QYJ collected important background information. WXW participated in the design and helped to draft the manuscript. All authors read and approved the final manuscript. 
Acknowledgements

We thank Helix (https://www.helixlife.cn/) for help in English grammar and fluency.

\section{References}

1. Bast RC, Hennessy B, Mills GBJNRC: The biology of ovarian cancer: new opportunities for translation. 2009, 9(6):415-428.

2. Cho KR, Shih le M: Ovarian cancer. Annual review of pathology 2009, 4:287-313.

3. Rethinking ovarian cancer II: reducing mortality from high-grade serous ovarian cancer \%J Nature Reviews Cancer.

4. Bell DA: Origins and molecular pathology of ovarian cancer. Modern pathology : an official journal of the United States and Canadian Academy of Pathology, Inc 2005, 18 Suppl 2:S19-32.

5. Seidman JD, Horkayne-Szakaly I, Haiba M, Boice CR, Kurman RJ, Ronnett BM: The histologic type and stage distribution of ovarian carcinomas of surface epithelial origin. International journal of gynecological pathology : official journal of the International Society of Gynecological Pathologists 2004, 23(1):41-44.

6. Kipps E, Tan D, Kaye SBJNRC: Meeting the challenge of ascites in ovarian cancer: new avenues for therapy and research.

7. Lengyel E: Ovarian cancer development and metastasis. The American journal of pathology 2010, 177(3):1053-1064.

8. Mc Mullen M, Karakasis K, Rottapel R, Oza AMJNC: Advances in ovarian cancer, from biology to treatment. 2021, 2(1):6-8.

9. Shukla AA, Rameez S, Wolfe LS, Oien N: High-Throughput Process Development for Biopharmaceuticals. Advances in biochemical engineering/biotechnology 2018, 165:401-441.

10. Govindarajan M, Wohlmuth C, Waas M, Bernardini MQ, Kislinger T: High-throughput approaches for precision medicine in high-grade serous ovarian cancer. Journal of hematology \& oncology 2020, 13(1):134.

11. Barrett T, Wilhite SE, Ledoux P, Evangelista C, Kim IF, Tomashevsky M, Marshall KA, Phillippy KH, Sherman PM, Holko M et al: NCBI GEO: archive for functional genomics data sets-update. Nucleic acids research 2013, 41(Database issue):D991-995.

12. Tomczak K, Czerwińska P, Wiznerowicz M: The Cancer Genome Atlas (TCGA): an immeasurable source of knowledge. Contemporary oncology (Poznan, Poland) 2015, 19(1a):A68-77.

13. Bi J, Bi F, Pan X, Yang Q: Establishment of a novel glycolysis-related prognostic gene signature for ovarian cancer and its relationships with immune infiltration of the tumor microenvironment. Journal of translational medicine 2021, 19(1):382.

14. Jin J, Li Y, Muluh TA, Zhi L, Zhao Q: Identification of CXCL10-Relevant Tumor Microenvironment Characterization and Clinical Outcome in Ovarian Cancer. Frontiers in genetics 2021, 12:678747. 
15. Zhang D, Li Y, Yang S, Wang M, Yao J, Zheng Y, Deng Y, Li N, Wei B, Wu Y et al: Identification of a glycolysis-related gene signature for survival prediction of ovarian cancer patients. Cancer medicine 2021.

16. Zheng J, Guo J, Zhu L, Zhou Y, Tong J: Comprehensive analyses of glycolysis-related IncRNAs for ovarian cancer patients. Journal of ovarian research 2021, 14(1):124.

17. Zheng J, Guo J, Zhang H, Cao B, Xu G, Zhang Z, Tong J: Four Prognosis-Associated IncRNAs Serve as Biomarkers in Ovarian Cancer. Frontiers in genetics 2021, 12:672674.

18. Su R, Jin C, Zhou L, Cao Y, Kuang M, Li L, Xiang J: Construction of a ceRNA network of hub genes affecting immune infiltration in ovarian cancer identified by WGCNA. BMC cancer 2021, 21(1):970.

19. Feng C, Xu Y, Liu Y, Zhu L, Wang L, Cui X, Lu J, Zhang Y, Zhou L, Chen M et al: Gene Expression Subtyping Reveals Immune alterations:TCGA Database for Prognosis in Ovarian Serous Cystadenocarcinoma. Frontiers in molecular biosciences 2021, 8:619027.

20. Liang L, Yu J, Li J, Li N, Liu J, Xiu L, Zeng J, Wang T, Wu L: Integration of scRNA-Seq and Bulk RNASeq to Analyse the Heterogeneity of Ovarian Cancer Immune Cells and Establish a Molecular Risk Model. Frontiers in oncology 2021, 11:711020.

21. Wang W, Liu Y, Yang Y, Huang X, Hou Y: MMP-16 as a New Biomarker for Predicting Prognosis and Chemosensitivity of Serous Ovarian Cancer: A Study Based on Bioinformatics Analysis. Critical reviews in eukaryotic gene expression 2021, 31(4):1-8.

22. Fan $\mathrm{L}$, Yang $\mathrm{H}$, Zhang B, Ding H: MCUR1 is a prognostic biomarker for ovarian cancer patients. Cancer biomarkers : section A of Disease markers 2021.

23. Xu H, Zou R, Li F, Liu J, Luan N, Wang S, Zhu L: MRPL15 is a novel prognostic biomarker and therapeutic target for epithelial ovarian cancer. Cancer medicine 2021, 10(11):3655-3673.

24. Davis S, Meltzer PS: GEOquery: a bridge between the Gene Expression Omnibus (GEO) and BioConductor. Bioinformatics (Oxford, England) 2007, 23(14):1846-1847.

25. Ritchie ME, Phipson B, Wu D, Hu Y, Law CW, Shi W, Smyth GK: limma powers differential expression analyses for RNA-sequencing and microarray studies. Nucleic acids research 2015, 43(7):e47.

26. Love MI, Huber W, Anders SJGB: Moderated estimation of fold change and dispersion for RNA-seq data with DESeq2. 2014, 15(12):550.

27. Mayakonda A, Koeffler HP: Maftools: Efficient analysis, visualization and summarization of MAF files from large-scale cohort based cancer studies. 2016.

28. Colaprico A, Silva TC, Olsen C, Garofano L, Noushmehr HJNAR: TCGAbiolinks: An R/Bioconductor package for integrative analysis of TCGA data. 2015, 44(8).

29. Coletta A, Molter C, Duqué R, Steenhoff D, Taminau J, Schaetzen VD, Meganck S, Lazar C, Venet D, Detours V: in GenePattern, Integrative Genomics Viewer, and R/Bioconductor. 2013.

30. Dileo MV, Strahan GD, Bakker M, Hoekenga OAJPO: Weighted Correlation Network Analysis (WGCNA) Applied to the Tomato Fruit Metabolome. 2011, 6. 
31. Ashburner M, Ball CA, Blake JA, Botstein D, Cherry JMJNG: Gene ontology: tool for the unification of biology. The Gene Ontology Consortium. 2000, 25(1):25-29.

32. Ogata H, Goto S, Sato K, Fujibuchi W, Kanehisa MJNAR: KEGG: kyoto Encyclopedia of Genes and Genomes. 1999, 27(1):29-34.

33. Yu G, Wang LG, Han Y, He QYJO-aJoIB: clusterProfiler: an R package for comparing biological themes among gene clusters. 2012, 16(5):284-287.

34. Suárez-Fari?As M, Lowes MA, Zaba LC, Krueger JG, Soyer HPJPO: Evaluation of the Psoriasis Transcriptome across Different Studies by Gene Set Enrichment Analysis (GSEA). 2012, 5(4):e10247.

35. Liberzon A, Birger C, Thorvaldsdóttir H, Ghandi M, Mesirov J, Tamayo PJCS: The Molecular Signatures Database (MSigDB) hallmark gene set collection. 2015, 1(6):417-425.

36. Reinhold WC, Sunshine M, Liu H, Varma S, Kohn KW, Morris J, Doroshow J, Pommier Y: CellMiner: a web-based suite of genomic and pharmacologic tools to explore transcript and drug patterns in the NCl-60 cell line set. Cancer research 2012, 72(14):3499-3511.

37. Shankavaram UT, Varma S, Kane D, Sunshine M, Chary KK, Reinhold WC, Pommier Y, Weinstein JN: CellMiner: a relational database and query tool for the $\mathrm{NCl}-60$ cancer cell lines. BMC genomics 2009, 10:277.

38. Yang W, Jorge S, Patricia G, Edelman EJ, Howard L, Simon F, Nidhi B, Dave B, Smith JA, Richard TIJNAR: Genomics of Drug Sensitivity in Cancer (GDSC): a resource for therapeutic biomarker discovery in cancer cells. 2013(D1):D955.

39. Paul G, Nancy C, Huang RS, Barbour JDJPO: pRRophetic: An R Package for Prediction of Clinical Chemotherapeutic Response from Tumor Gene Expression Levels. 2014, 9(9):e107468-e107468.

40. Newman AM, Liu CL, Green MR, Gentles AJ, Feng W, Xu Y, Hoang CD, Diehn M, Alizadeh AA: Robust enumeration of cell subsets from tissue expression profiles. Nature methods 2015, 12(5):453-457.

41. Sato E, Muto J, Zhang LJ, Adase CA, Sanford JA, Takahashi T, Nakatsuji T, Usdin TB, Gallo RL: The Parathyroid Hormone Second Receptor PTH2R and its Ligand Tuberoinfundibular Peptide of 39 Residues TIP39 Regulate Intracellular Calcium and Influence Keratinocyte Differentiation. The Journal of investigative dermatology 2016, 136(7):1449-1459.

42. Lheureux S, Gourley C, Vergote I, Oza AM: Epithelial ovarian cancer. Lancet (London, England) 2019, 393(10177):1240-1253.

\section{Figures}




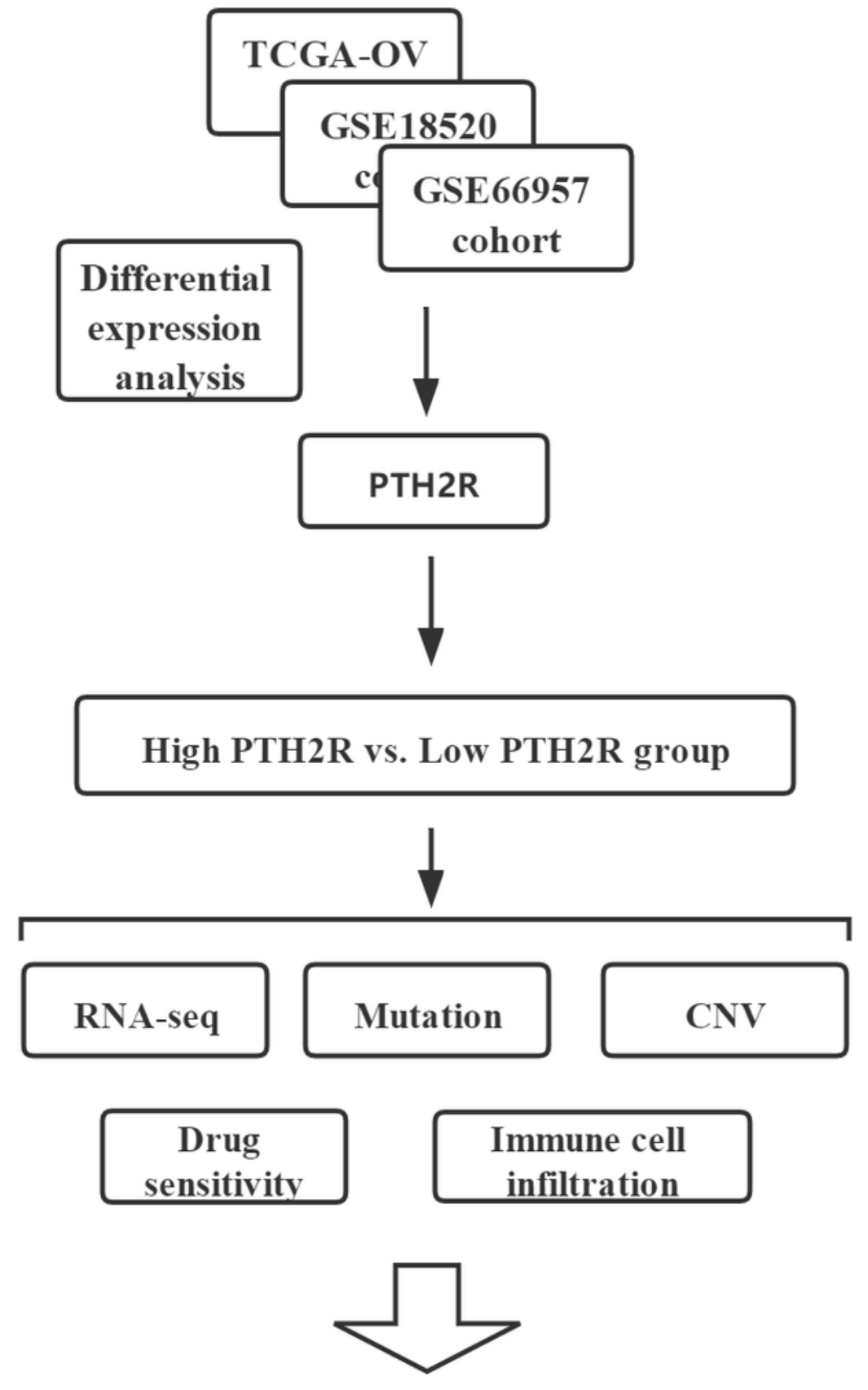

Experiments: PTH2R expression and function verification

Figure 1

The visual flow-process diagram of this study. 
A

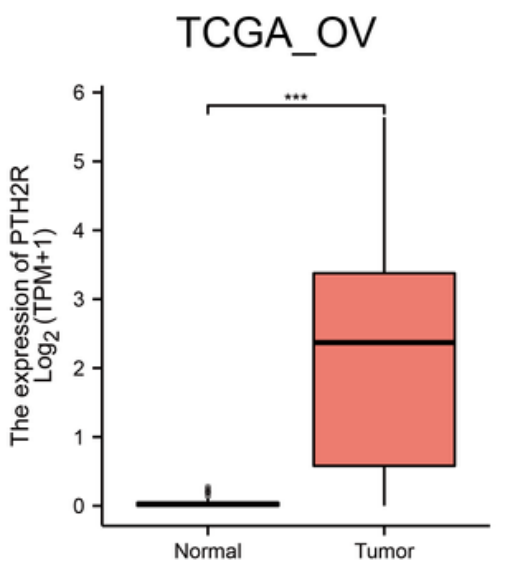

B

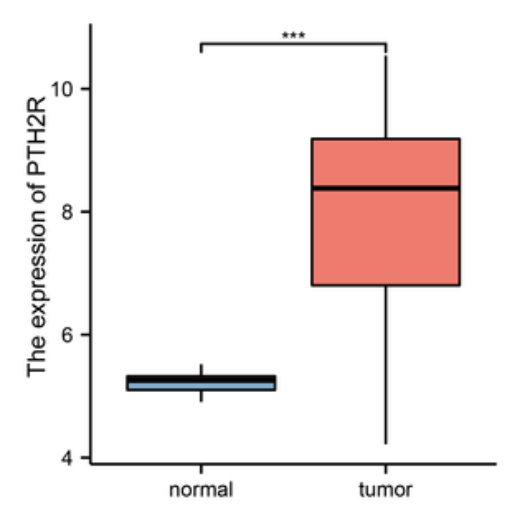

D

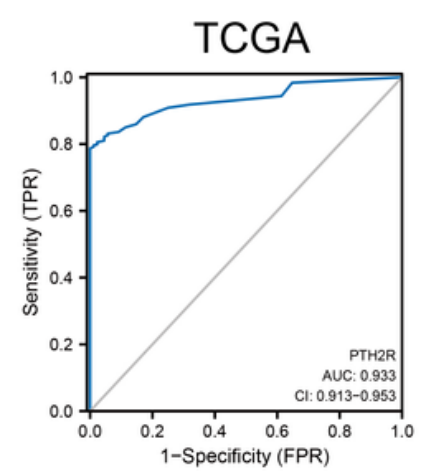

$\mathrm{E}$

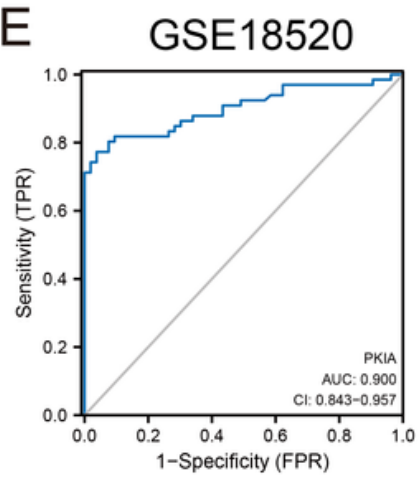

$\mathrm{H}$

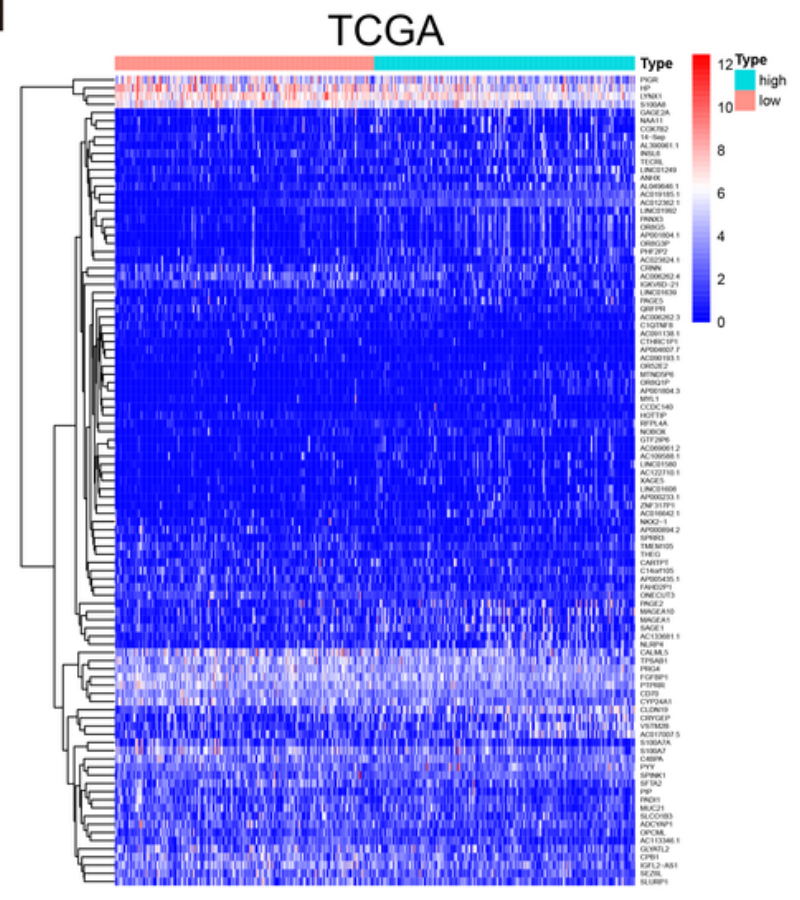

C
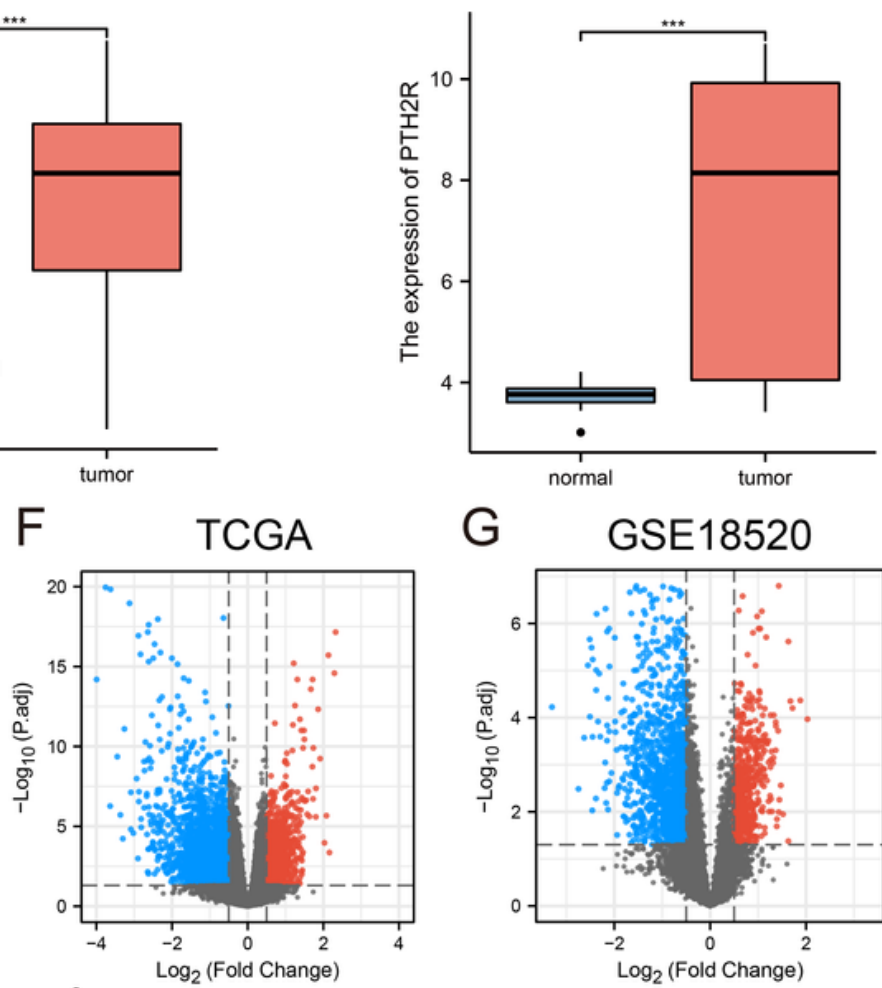

G GSE18520

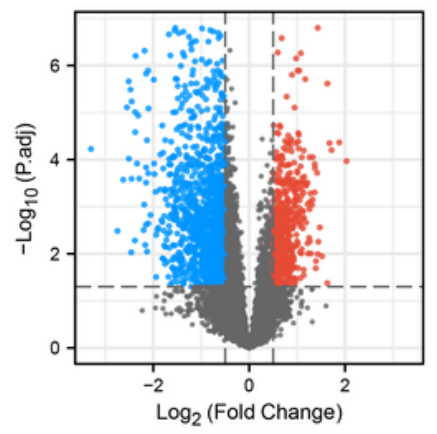

I

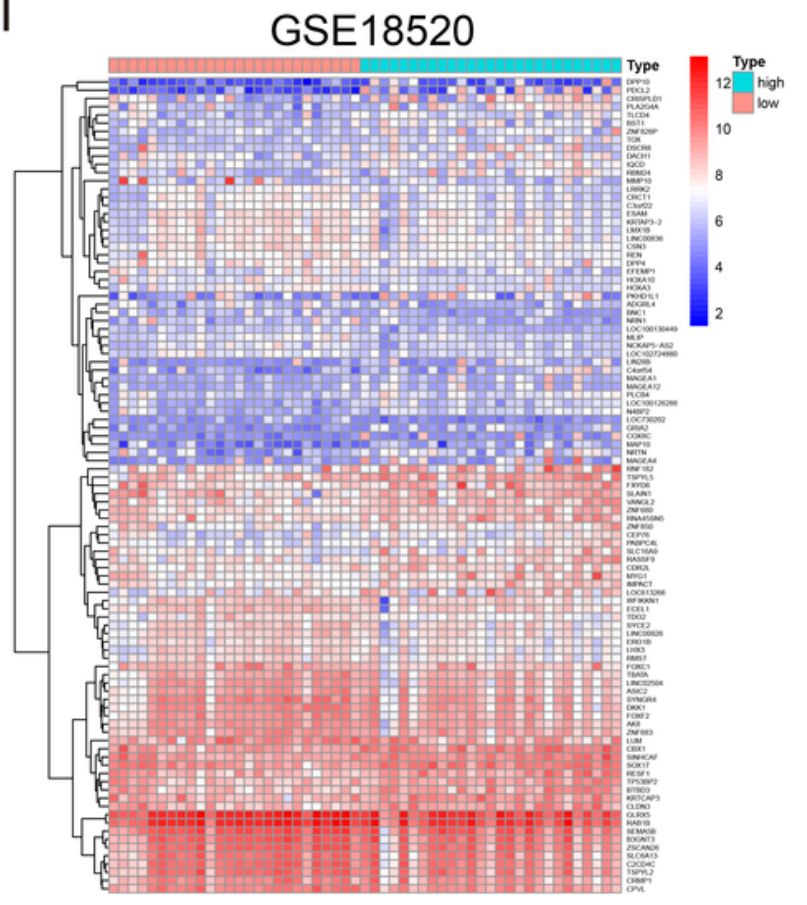

Figure 2

PTH2R is overexpressed in tumor. A. The TCGA_OV dataset revealed that PTH2R was highly expressed in tumor tissues. B. The GSE18520 dataset revealed that PTH2R was highly expressed in tumor tissues. C. The GSE66957 dataset revealed that PTH2R was highly expressed in tumor tissues. D-E. The ROC curve of TCGA_OV and GSE18520 dataset according to the expression of PTH2R. F-I. The volcano plots and heatmaps showed the differentially expressed genes in TCGA_OV and GSE18520. 
A

PTH2R_high

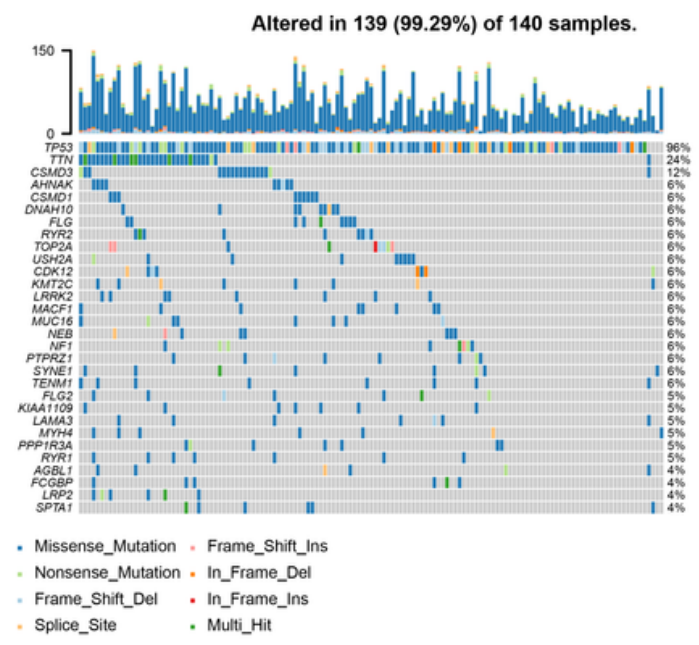

C

$\left.\begin{array}{l}5 \\ \\ 1\end{array}\right]$

PTH2R : [Somatic Mutation Rate: $0.46 \%$ ] NM_005048

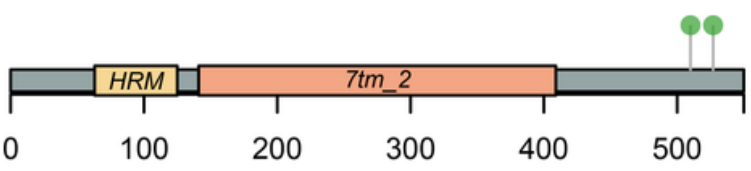

B

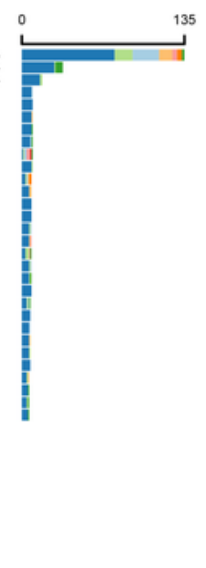

$\mathrm{D}$

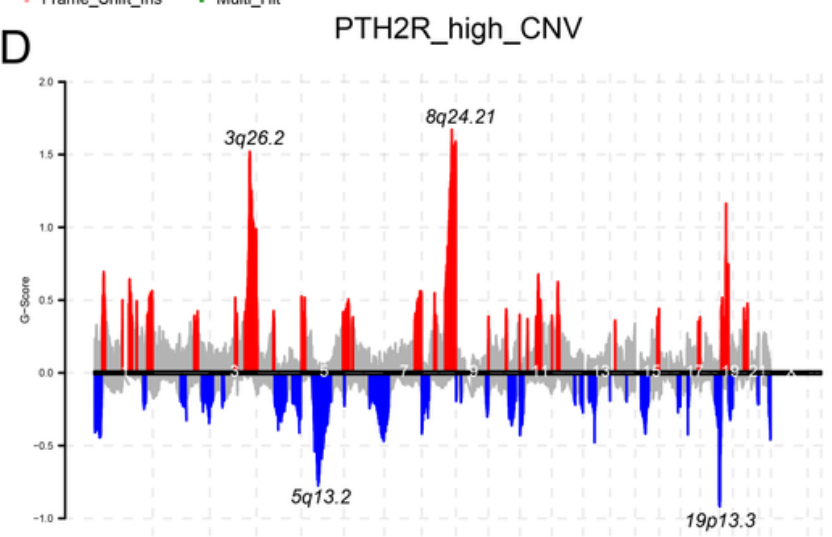

\section{Figure 3}

The alteration of mutations and copy number variation in high PTH2R expression group and low PTH2R expression group. A. The top 30 mutation genes in high PTH2R expression group. B. The top 30 mutation genes in low PTH2R expression group. C. The mutation sites information of PTH2R. D. The CNV in the PTH2R high expression group. 
A

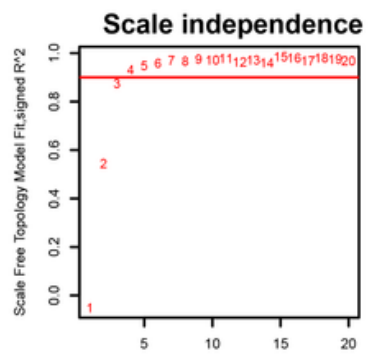

B

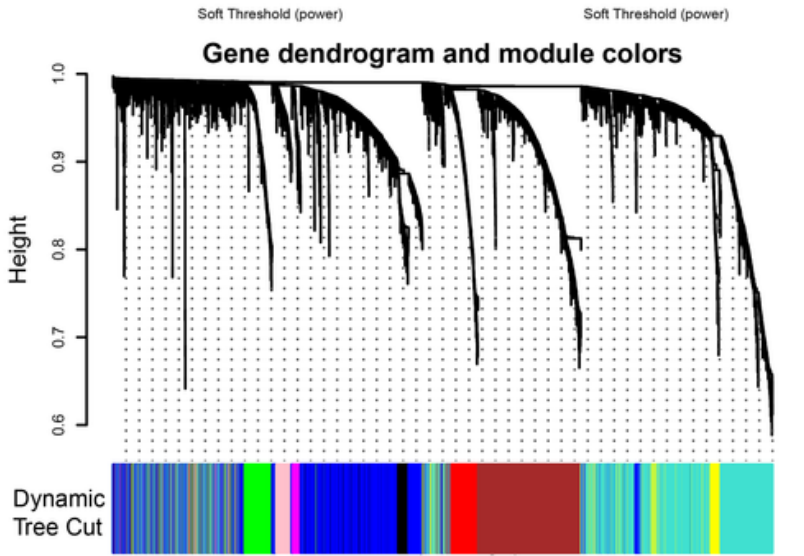

D

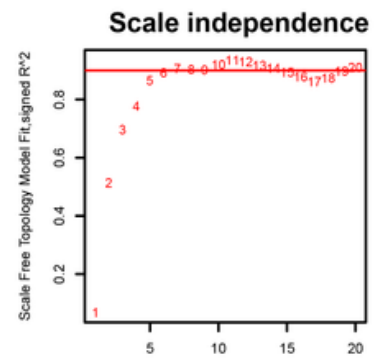

$E$
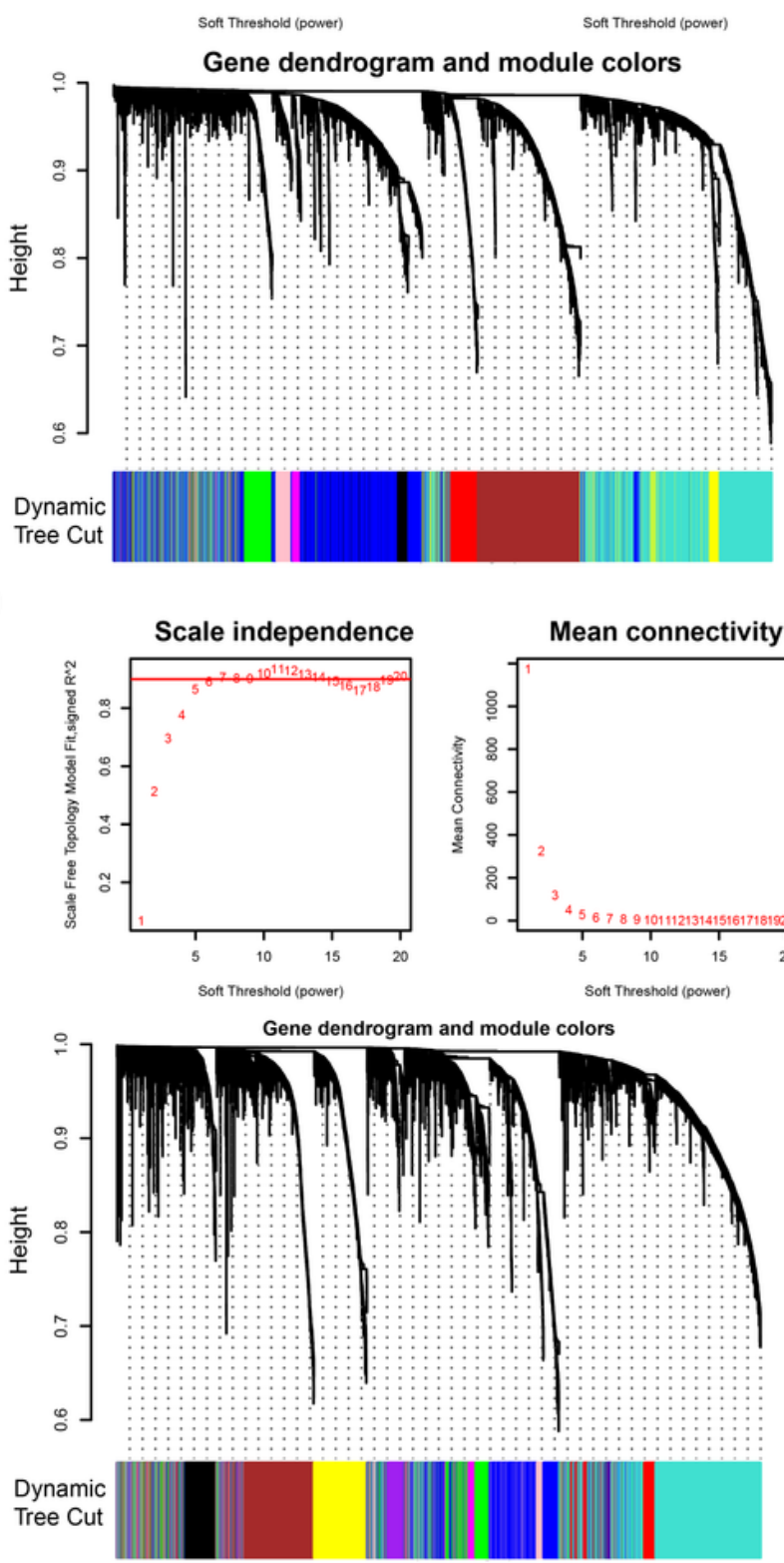

C
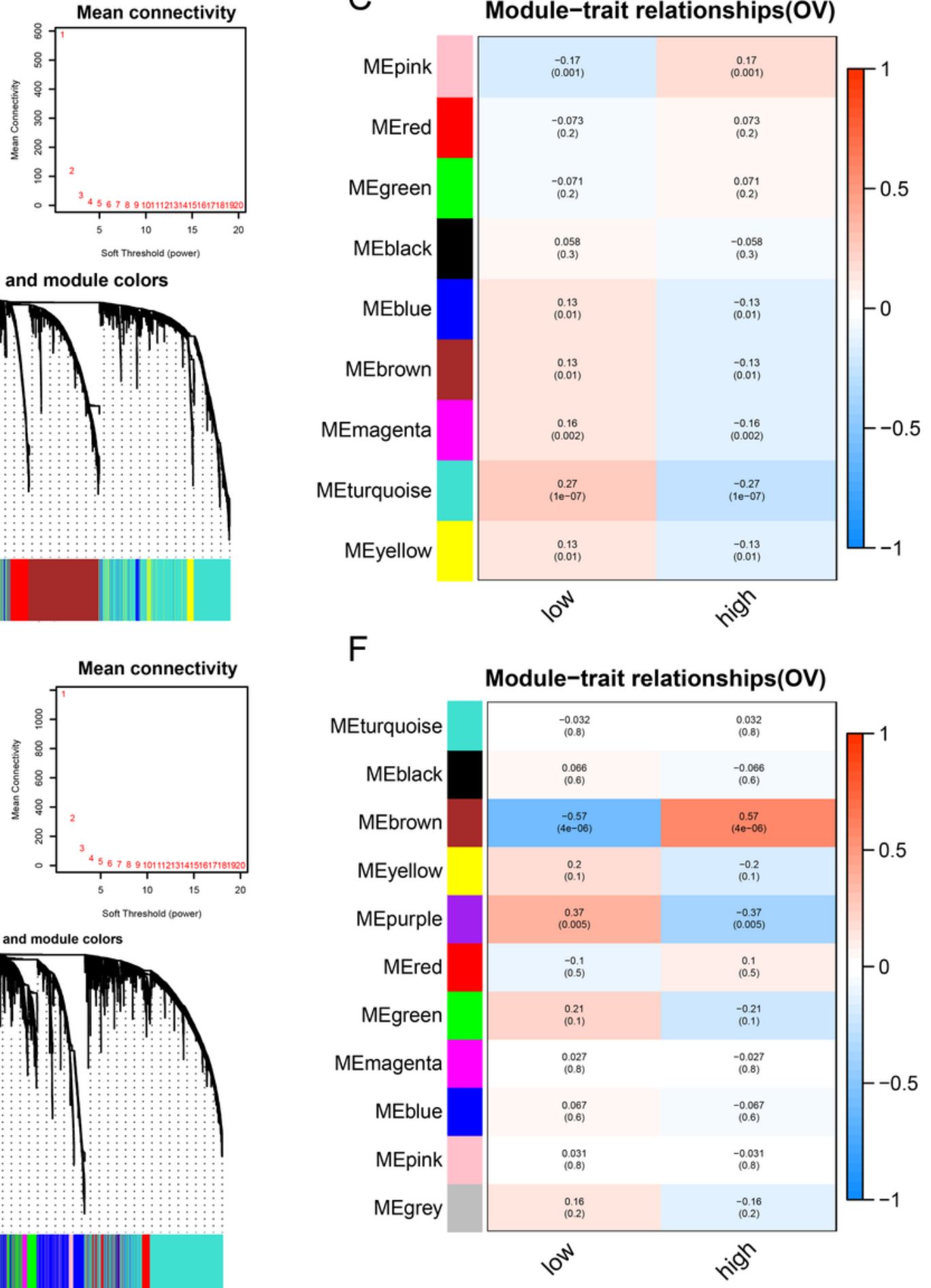

Figure 4

WGCNA analysis. A. The lowest power for which scale independence in TCGA_OV dataset. B. Repeated hierarchical clustering tree. $\mathrm{C}$. The associations between phenotypes and the modules in TCGA_OV dataset. E. The lowest power for which scale independence in GSE18520 dataset. F. Repeated hierarchical clustering tree in GSE18520. G. The associations between phenotypes and the modules in GSE18520 dataset. 
A

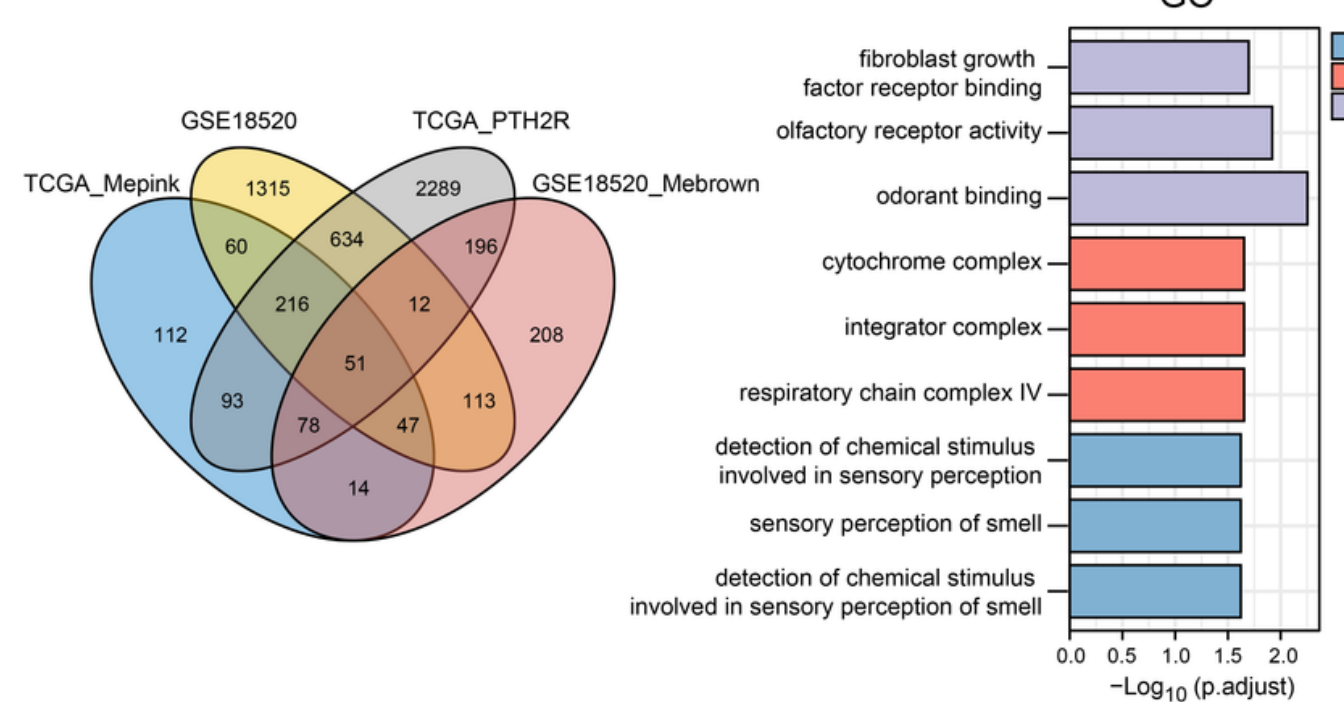

$\mathrm{D}$

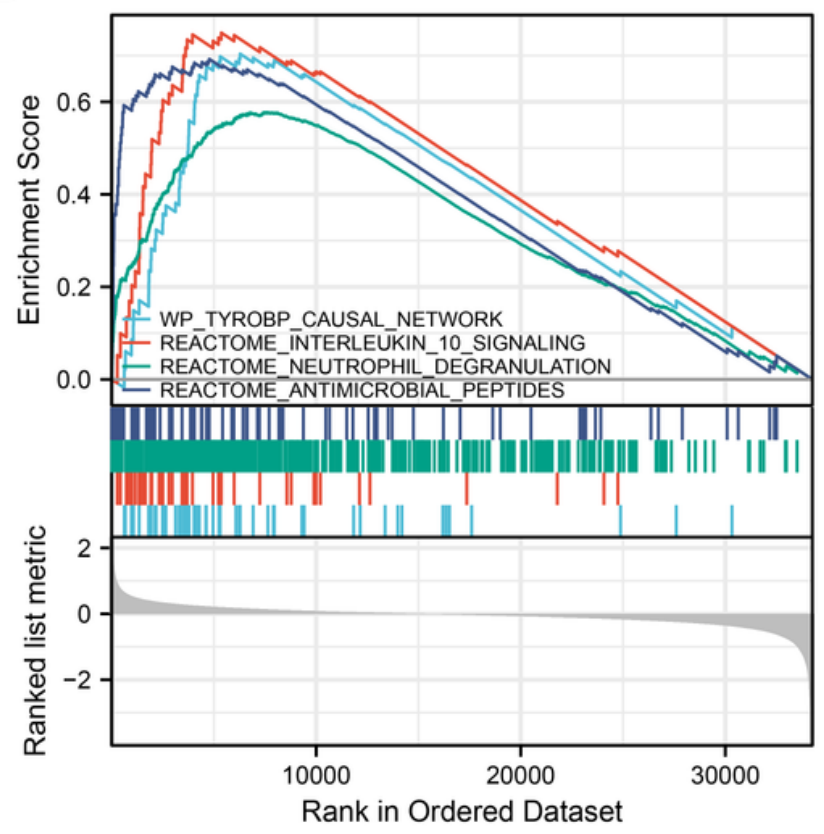

B

$\mathrm{E}$

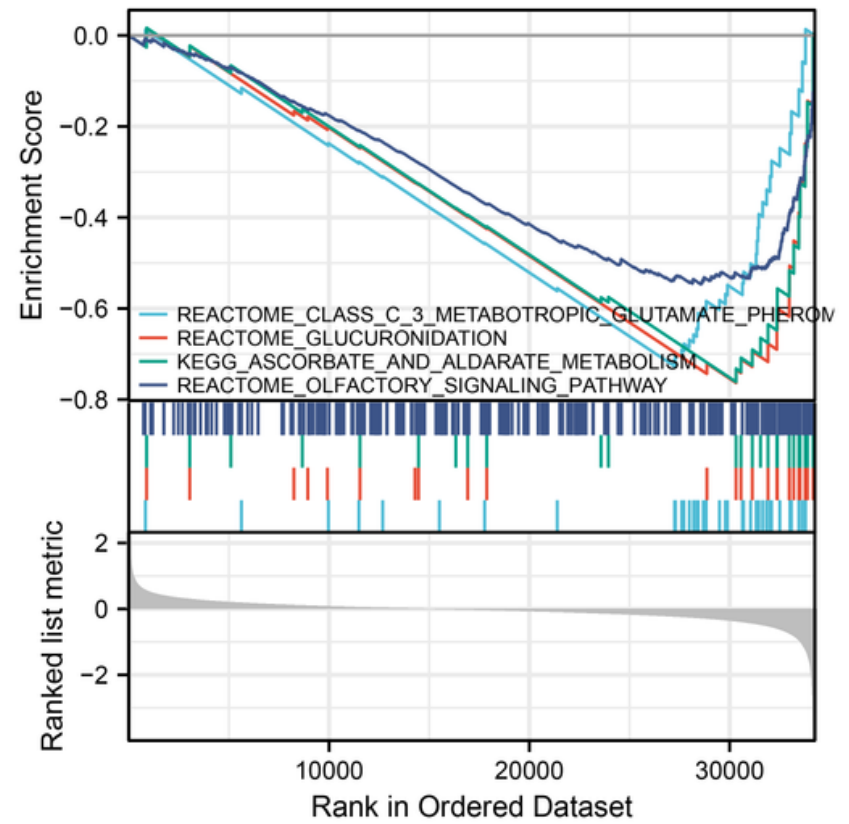

Figure 5

Enrichment analysis. A. The Venn diagram of DEGs and WGCNA candidate genes. B-C. GO and KEGG pathway enrichment of candidate 51 candidate genes. D. the significant enriched pathway in high PTH2R expression with GSEA. E. the significant enriched pathway in low PTH2R expression with GSEA. 
A

PTH2R, Epothilone B Cor $=-0.567, p<0.001$

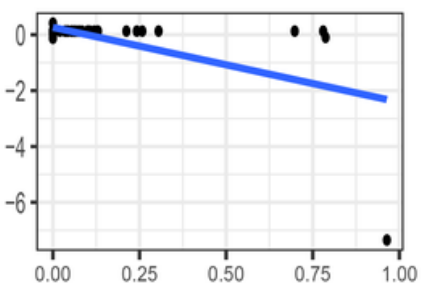
PTH2R, Actinomycin D Cor $=-0.364, p=0.004$

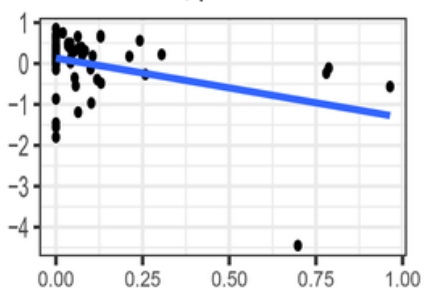

B
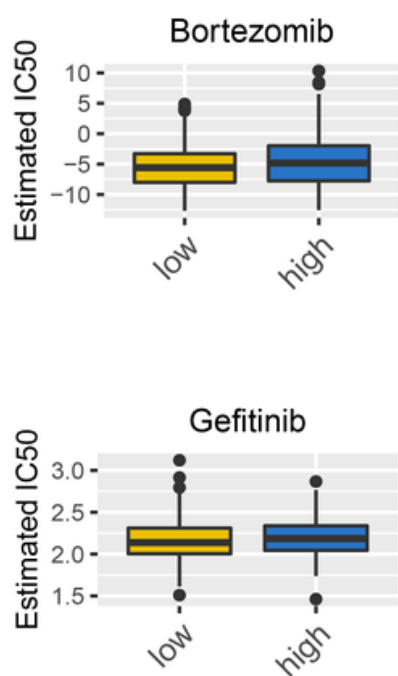

PTH2R, Alvespimycin Cor $=-0.427, p<0.001$

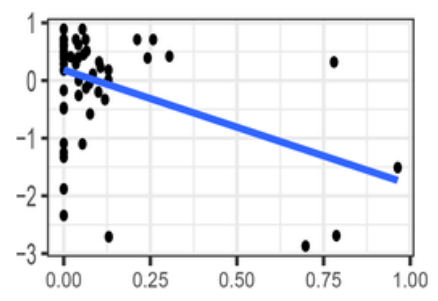

PTH2R, Mithramycin Cor $=-0.315, p=0.014$

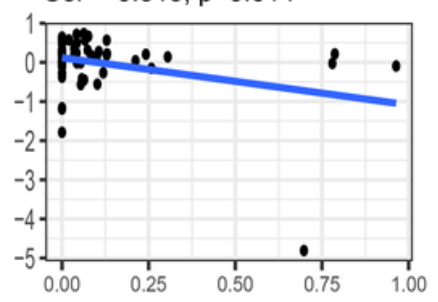

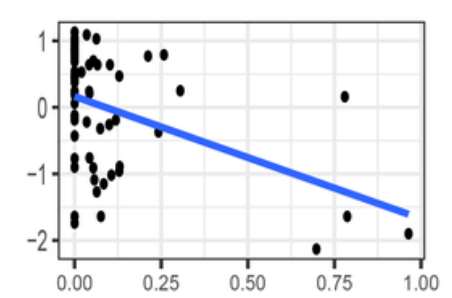

PTH2R, Depsipeptide Cor $=-0.305, p=0.018$

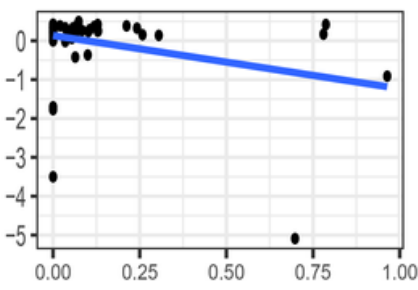

PTH2R, Tanespimycin Cor $=-0.426, p<0.001$

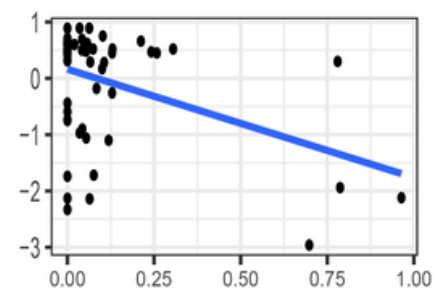

PTH2R, Pelitrexol

Cor $=-0.301, p=0.019$

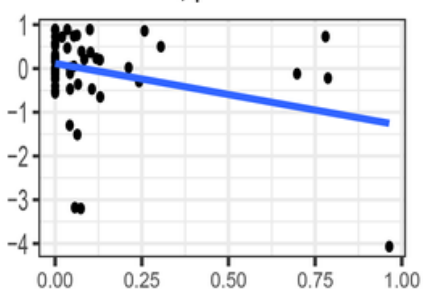

PTH2R, geldanamycin analog Cor $=-0.403, p=0.001$ 
A

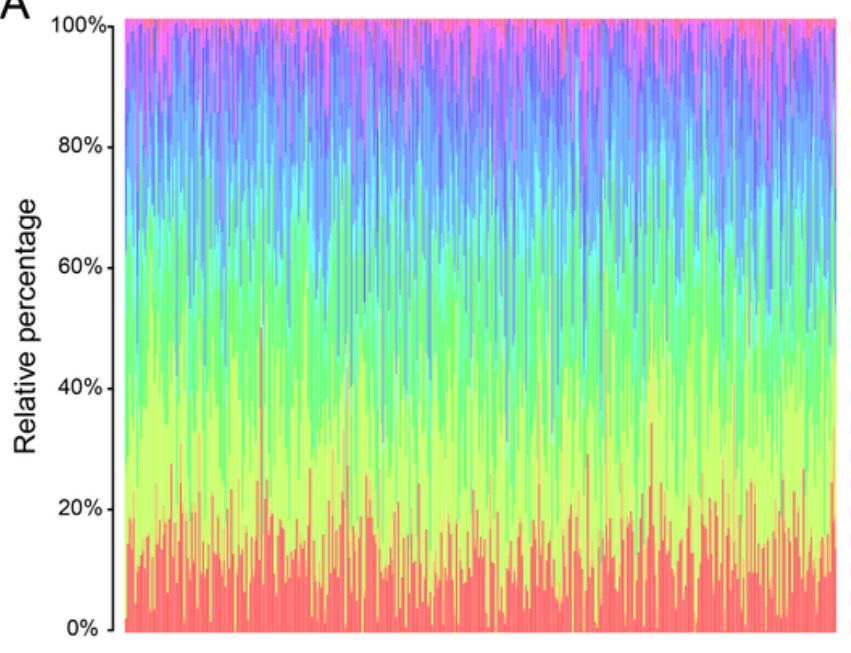

B cells naive

- $B$ cells memory

Plasma cells

T cells $\mathrm{CD} 8$

T cells CD4 naive

T cells $C D 4$ memory resting

T cells CD4 memory activated

- T cells follicular helper

- T cells regulatory (Tregs)

- T cells gamma delta

NK cells resting

NK cells activated

Monocytes

Macrophages MO

Macrophages M1

Macrophages M2

Dendritic cells resting

- Dendritic cells activated

Mast cells resting

- Mast cells activated

Eosinophils

Neutrophils

C

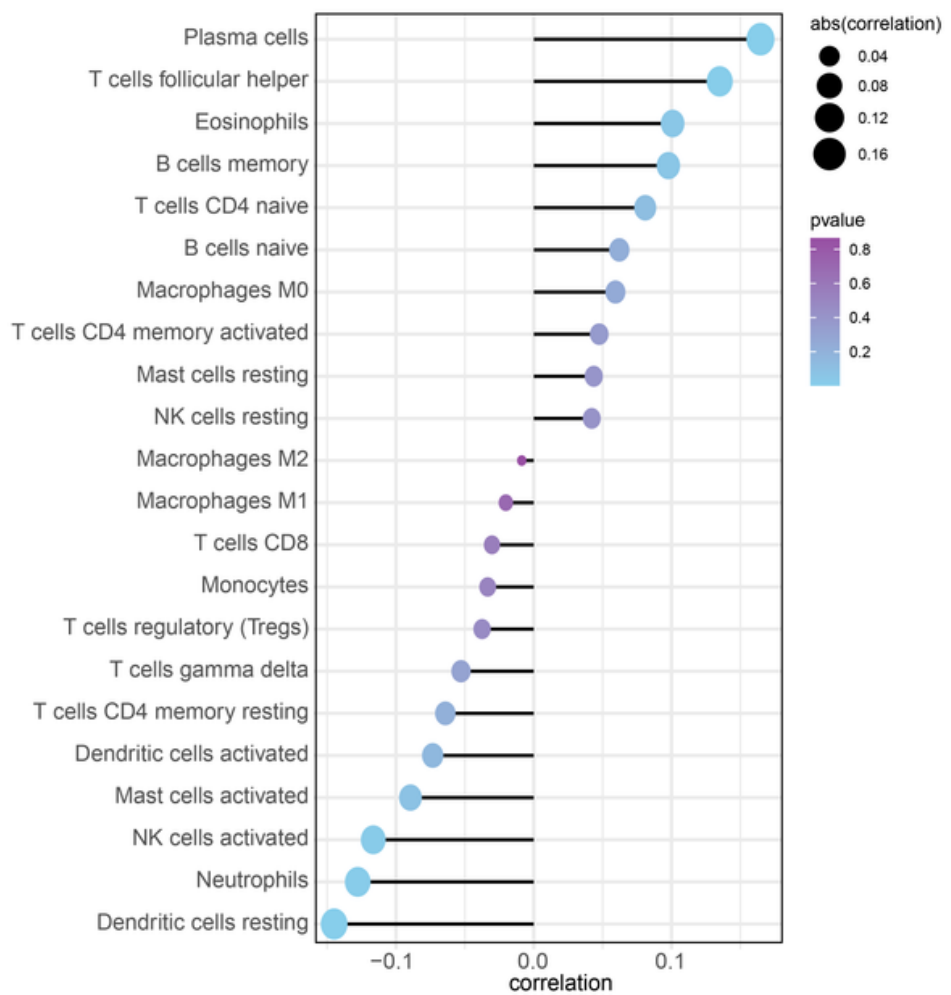

B

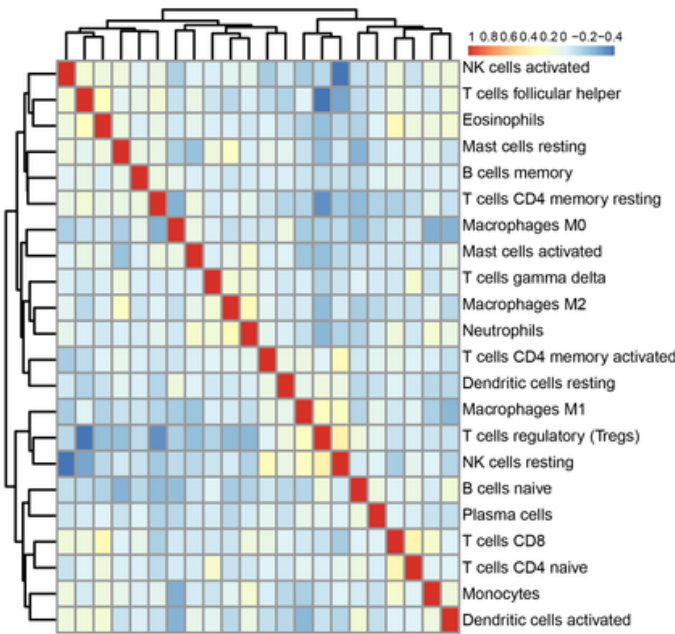

D
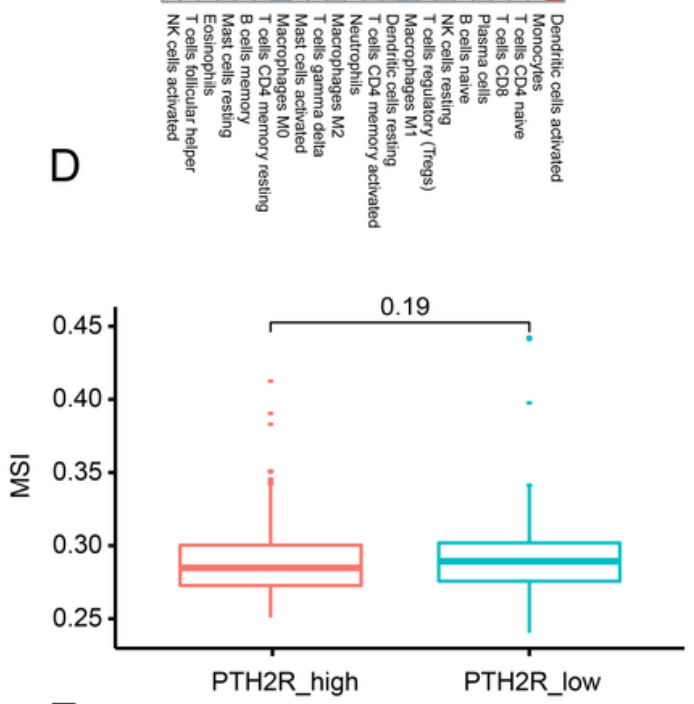

E

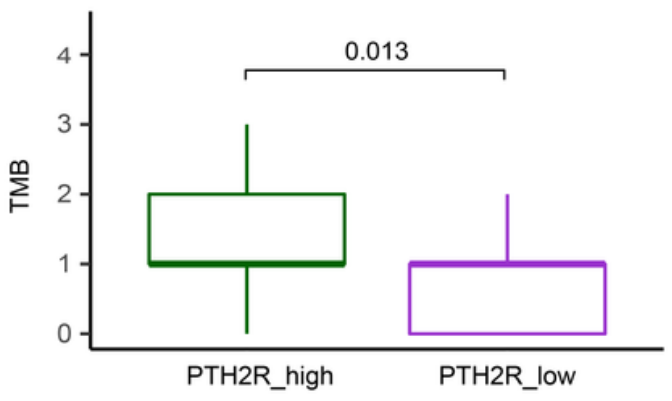

\section{Figure 7}

The landscape of immune microenvironment in TCGA_OV and PTH2R related immune cells correlation. A-B. The landscape of immune microenvironment in TCGA_OV and correlation between 22 immune cell infiltration score. C. The correlation between PTH2R expression and infiltrated immune cells. D-E. The difference between MSI and TMB in PTH2R high expression and low expression group. 
A

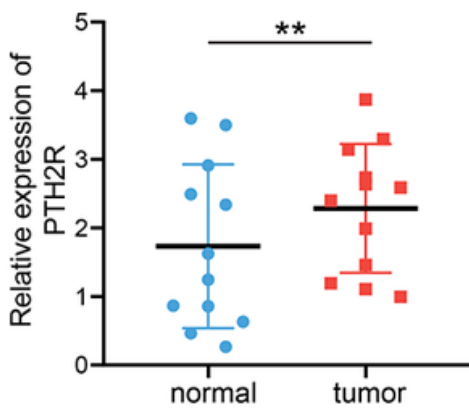

D

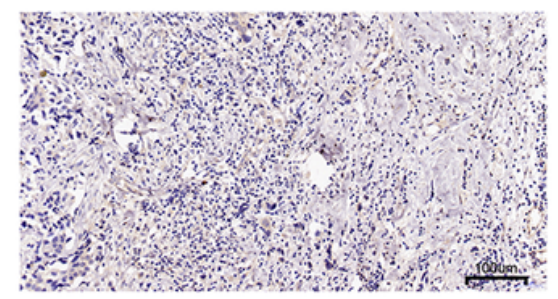

T1

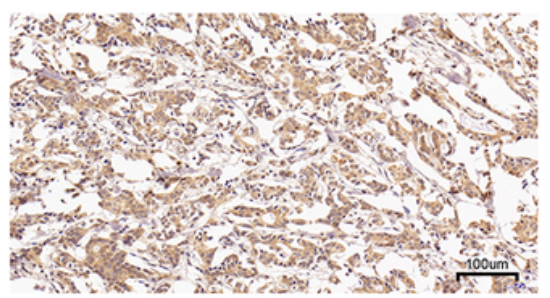

B

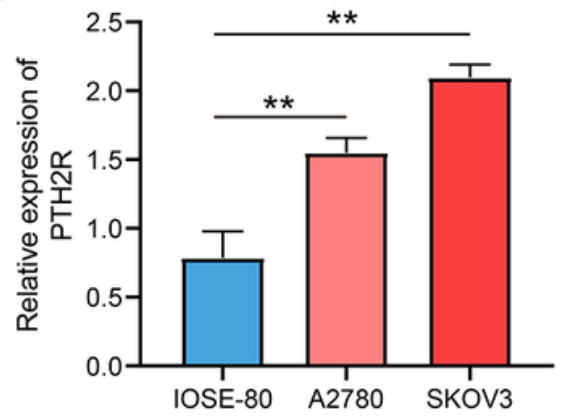

N2

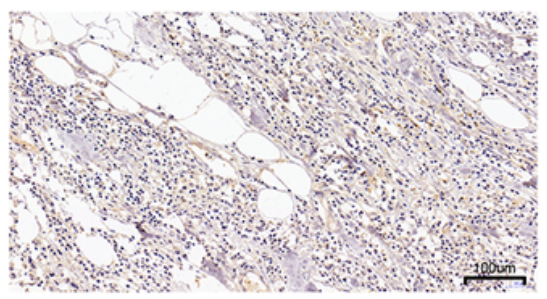

T2

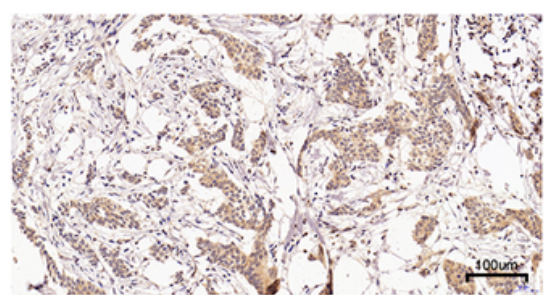

C

$\begin{array}{llllll}\mathrm{T} 1 & \mathrm{~N} 1 & \mathrm{~T} 2 & \mathrm{~N} 2 & \mathrm{~T} 3 & \mathrm{~N} 3\end{array}$

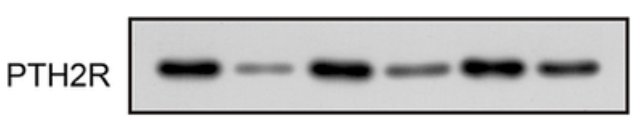

GAPDH

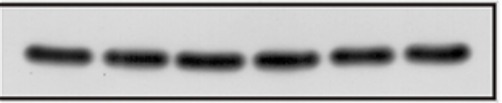

N3

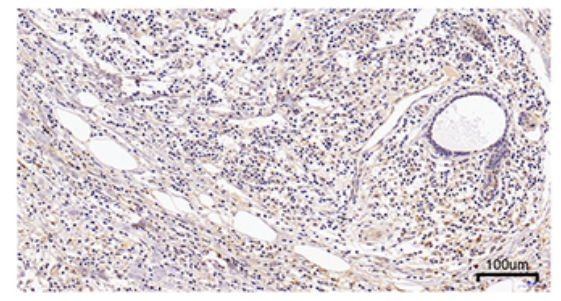

T3

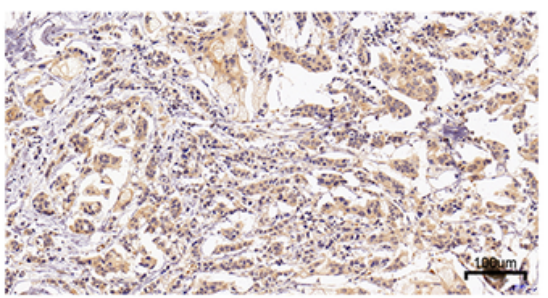

\section{Figure 8}

Validation of PTH2R expression in tissues and cells. A-B. The qPCR showed that PTH2R is highly expressed in tumor tissues and cells. B-C. The western blot and IHC showed that PTH2R protein is highly expressed in tumor tissues. ${ }^{*} p<0.05,{ }^{\star *} p<0.01$. 
A

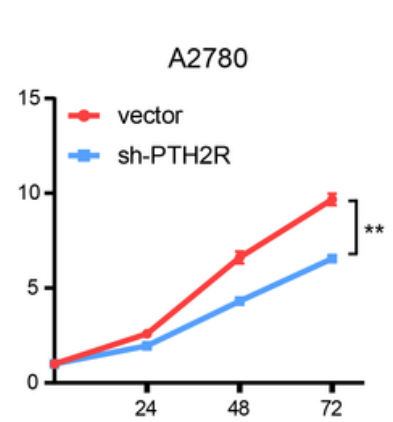

D

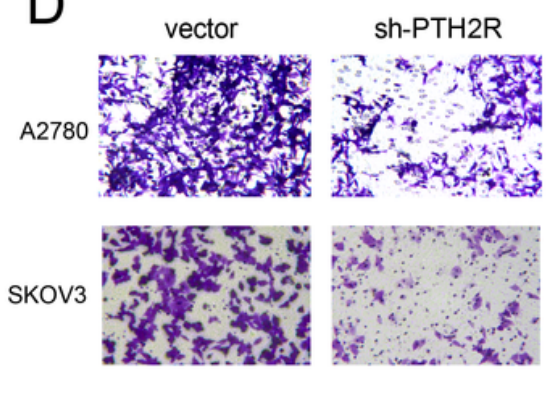

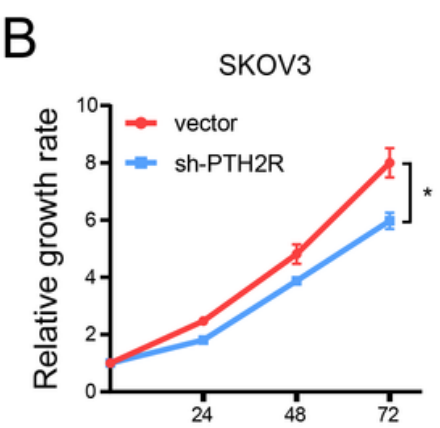

C

A2780

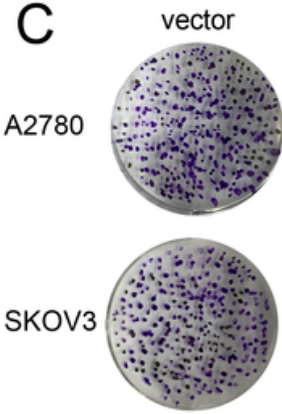

$E$
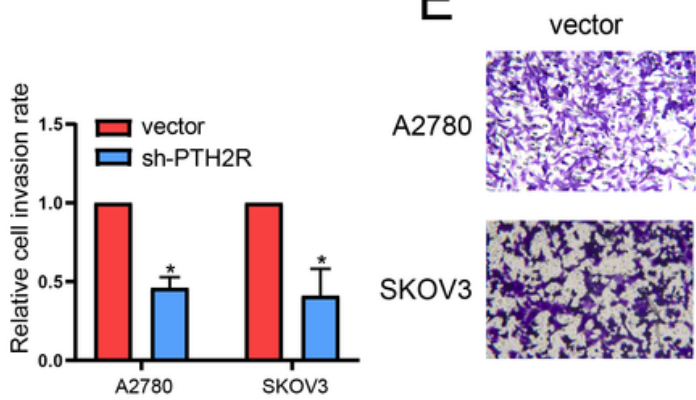
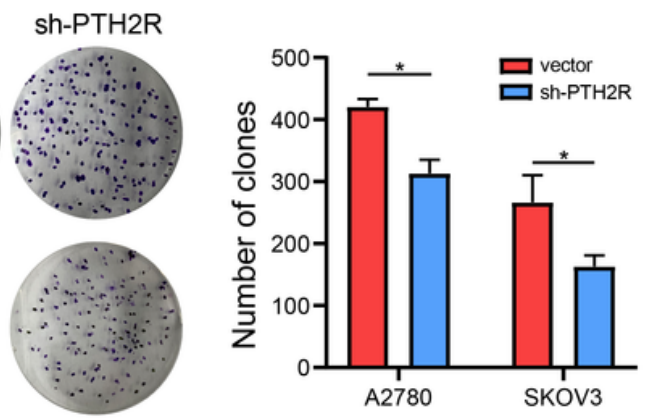
sh-PTH2R
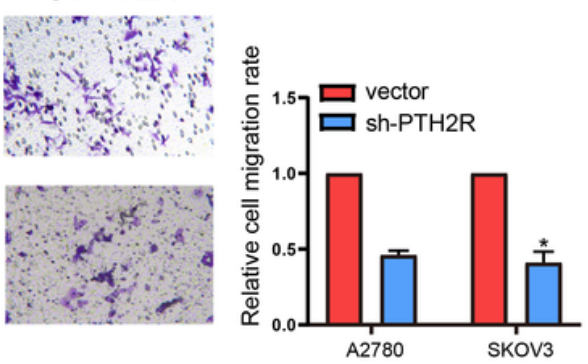

\section{Figure 9}

High expressed PTH2R is associated tumor proliferation, invasion and migration. A-C. CCK-8 and clone formation assays showed that down regulation of PTH2R expression inhibited the proliferation of SKOV3 and A2780 cells. D-E. Transwell assay showed that down regulation of PTH2R expression inhibited the invasion and migration of SKOV3 and A2780 cells. 